\title{
A Multi-Criteria Cataloging of the Immovable Items of Industrial Heritage of Andalusia
}

\author{
Juan Claver ${ }^{1, *(\mathbb{D}}$, Amabel García-Domínguez ${ }^{1}$, Lorenzo Sevilla ${ }^{2} \mathbb{\text { and }}$ aiguel A. Sebastián ${ }^{1}$ \\ 1 Department of Construction and Manufacturing Engineering, Universidad Nacional de Educación a \\ Distancia (UNED), C/Juan del Rosal 12, 28040 Madrid, Spain; amabel.garcia@invi.uned.es (A.G.-D.); \\ msebastian@ind.uned.es (M.A.S.) \\ 2 Department of Civil, Materials and Manufacturing Engineering. Universidad de Málaga (UMA), \\ C/ Dr. Ortiz Ramos, 29071 Málaga, Spain; lsevilla@uma.es \\ * Correspondence: jclaver@ind.uned.es; Tel.: +34-91-398-60-88
}

Received: 31 October 2018; Accepted: 10 January 2019; Published: 14 January 2019

\begin{abstract}
Any research in any field needs an initial background, and in the same way, any decision should be supported by previous knowledge and study of the problem and its context. In the case of the industrial heritage, both the study of the typology and the decision making about the actions of conservation and reutilization of its assets must be based on a deep knowledge of the set of elements that the typology includes. All of that refers to the corresponding territory being analyzed, since the intensity and productive tradition will be different between each territory, region, or country. In that context, this paper represents the continuation of the main research line of the authors, and exposes their efforts to develop a useful tool for the study, management, and cultural promotion of the assets related to industrial heritage in Spain through the development of a multi-criteria catalogue of assets. Thus, based on the initial catalogue developed by some of the authors, this paper significantly increases the number of assets considered. In addition, it includes new classification criteria, reviews the observed trends, and establishes the future lines of work and suitable strategies for these kinds of initiatives.
\end{abstract}

Keywords: industrial heritage; cataloguing; methodology; multi-criteria; Andalusia; Spain

\section{Introduction}

In 2016, and as a part of a doctoral thesis, some of the authors elaborated a catalogue of immovable assets of the Spanish industrial heritage [1]. Despite the main objective of the research work being the design of a methodology based on multi-criteria decision-making tools, previous tasks that were identified included both the need of a wider catalogue of assets and a structure of classification criteria that applied to all of them. At that moment, the Initial Catalogue of the National Plan of Industrial Heritage [2], the Minimum Catalogue of the The International Committee for the Conservation of the Industrial Heritage (TICCIH)-Spain [3], and the catalogue developed by The DOCOMOMO (international committee for DOcumentation and COnservation of buildings, sites and neighborhoods of the MOdern MOvement) Foundation about the industrial buildings of the modern movement in Spain and Portugal [4], were the main cataloguing initiatives in the country. All of them were of great interest, especially with regard to their different approaches to the problem, but the number of assets that was considered in all of the cases was not large enough to be representative of this typology across the whole national territory.

The need to identify and classify the existing assets of value is considered in some of the most recognized documents about protection and actuation regarding the immovable assets of the cultural heritage, such as The Declaration of Amsterdam [5], which is focused on general architectural heritage, 
and The Niznhy Tagil Charter for the Industrial Heritage, which is specifically focused on this typology [6]. However, traditional cataloguing strategies must be revised and improved, both from a general approach to cultural heritage as well as from particular approaches to specific typologies $[7,8]$, as the industrial heritage is [9].

Unfortunately, it is not difficult to identify examples of reuse actions above these kinds of assets in which the relation to the productive process has disappeared. These situations reveal that the concept of industrial heritage and the real value of these assets are not yet sufficiently clear. In that sense, some of the widely recognized definitions describe the industrial heritage as the result of the relation between a social model, the capitalism, and the productive resources of the mechanization $[2,3]$. This approach involves chronological limits that have been discarded by the authors of this work, who have considered both preindustrial and industrial assets within the catalogue. It is the singularity with regard to aspects such as the technological exclusivity or innovation, the layout and sizing of the productive spaces, the structure or the constructive techniques applied in order to response to the needs of the productive process, or the historical and social role that the activity had in the region, which are the kinds of features that can provide cultural value to these kind of assets [1].

Thus, in the case of industrial heritage, the recording of these assets should enable the understanding of their productive nature from different approaches [10]. In that context, a new catalogue was developed, including 1354 assets that were classified through 54 different criteria. This made it possible to propose a great amount of analyses by combining different criteria in each case. However, some improvement goals have been identified since then.

From a general approach to the initial version of the catalogue of assets, it is obvious that although the number of assets that was considered was significantly higher than the sets of elements, including those referred to in the previous catalogues, there are many more assets of interest that were not identified in that initial version. In addition, the national approach of that first experience required careful work regarding the identification and selection of assets, avoiding any kind of focus on particular territories in order to show real trends. Although this effort developed the analysis of the presence of the typology in a horizontal way through the whole country, it also made it more difficult to address a complete identification of assets. Thus, after the achievement of the initial goal of having a more useful set of assets and a criteria structure applied to all of them, the own needs of this new tool began to appear.

The review of the identification and selection process on the different regions was the first aspect that was identified as a new goal. As in the first attempt, the catalogue needed to be representative of the presence of the typology in Spain, so it is important to avoid any kind of extra effort in the identification of assets in any particular territory. Controlling this through a unique revision above the whole catalogue can be difficult. In addition, considering the number of assets included, reviewing the whole set is a tedious task itself. Therefore, the authors considered the development of subsequent studies focused on particular Autonomous Communities or provinces as the most reasonable strategy for this review process. Thus, some previous review works were developed in territories as the provinces of Vigo, Cádiz, and Huelva [11-13], and in the Autonomous Community of the region of Murcia [14]. All of these works enabled comparisons between the initially obtained trends and the new ones, and at the same time, during their development, some clues about new needs, approaches, and solutions were obtained.

In this context, the whole revision of the initial version of the developed catalogue will be the result of a set of partial review works focused on particular territories. In that sense, this work represents the first significant review experience for the catalogue, since it affects a particularly important territory: the Autonomous Community of Andalusia. This territory has some special characteristics, such as its high number of provinces, the variety of industrial sectors and their different presence through the eight provinces, or the high number of industrial assets identified in this territory in the initial version of the catalogue. All of these reasons make Andalusia one of the best territories for showing the potential of the catalogue as a work tool, and it was also the selected territory when the initial 
version of the catalogue was presented to researchers in this field [15]. This way, this new work is understood as a key previous step to reviewing the catalogue at the national level.

In addition to the classification criteria, the developed catalogue includes complementary information, such as web entries and geolocation information for each element listed. Thus, for all of the new assets that were added to the catalogue, geolocation information was included, too. This data field has a special value, because in some cases, the location of these assets is little known given the remote location of many of them. Industrial assets are often abandoned when productive activity ends. Sometimes, even the residential settlements that are the result of these productive activities disappear when production ends. Since the dissemination and promotion of this typology and its assets have a key role in achieving their protection, the geolocation information of a large amount of assets provided a great opportunity for promoting their visit [16]. However, the geolocation of these assets is also important in research contexts, such as for example the implementation of geographic information systems in the study of these elements $[17,18]$. Figure 1 shows a map generated using Google My Maps. Introducing in this application the data of the columns of the catalogue, which contains the name given to each asset and the geolocation information included for each asset, these kind of maps are automatically created.

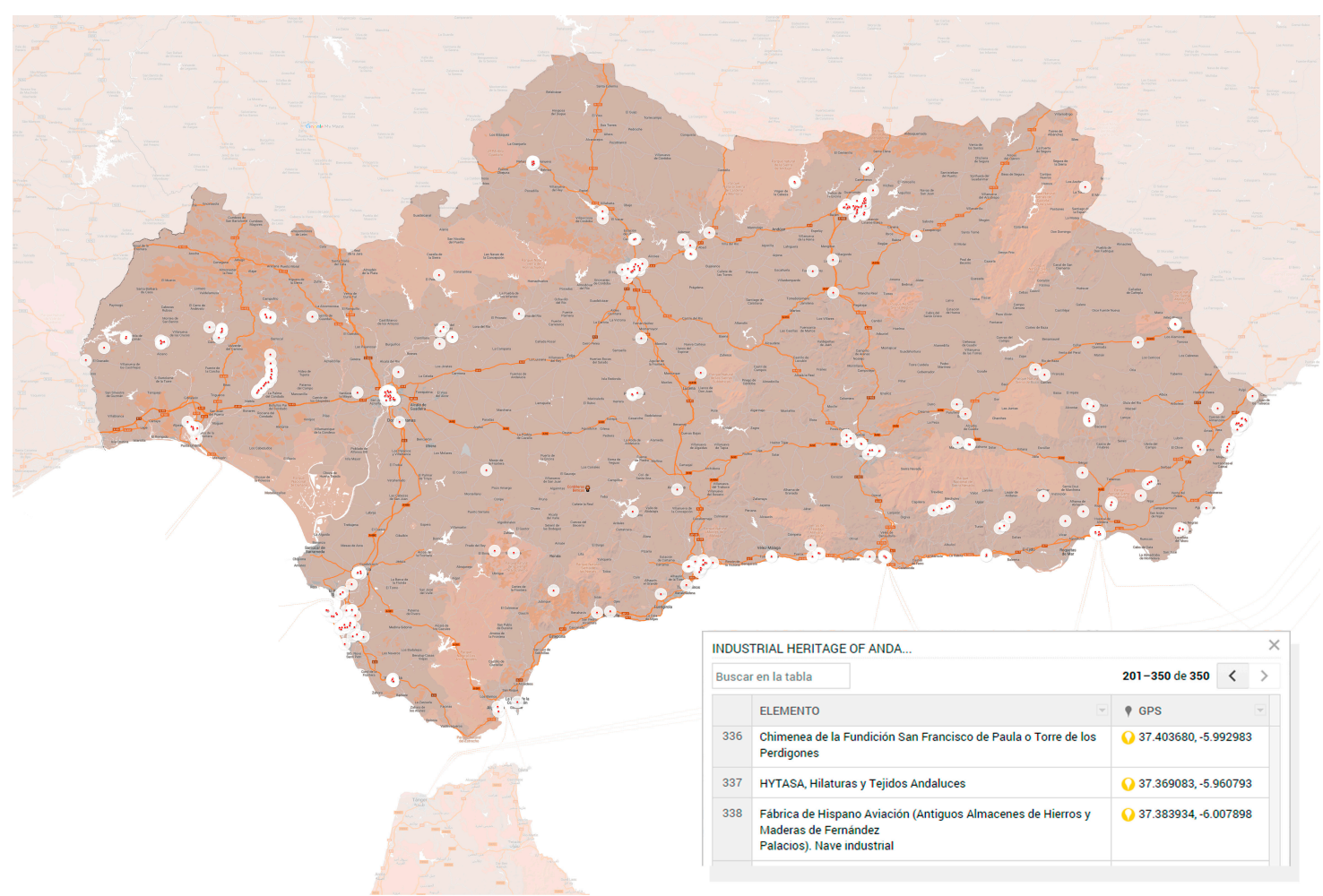

Figure 1. Visualization of the assets identified in Andalusia within a map created using Google My Maps and thanks to the geolocation information included for each asset in the catalogue.

Moreover, as it was previously introduced, the catalogue was born as support for the multi-criteria structures designed for the management and decision making about the reutilization of this type of assets, operating as one of the parts of a global methodology [19]. This methodology included three levels. Two of them were focused on the application of multi-criteria decision techniques, both for the valuation of the cultural interest of the analyzed assets and for the selection of the more compatible new activities. However, any decisions within these two levels had to be supported by a deep knowledge of this typology. In that context, a first version of a wide catalogue of industrial assets located in Spain was developed. In addition to the large number of assets considered in comparison to previous initiatives [2-4], the main characteristic of this catalogue was the application of a wide structure 
of classification criteria. This structure was applied to the whole set of assets, and by combining different classification criteria, it was possible to make a great variety of analyses of great interest in order to characterize this typology in Spain. Thus, the developed catalogue represents the necessary groundwork for those other criteria structures focused on multi-criteria decision-making, but these kinds of techniques are not applied within the catalogue.

During the operation of this methodology as a management tool, some new potential information fields of interest were identified. This work responses to these needs, and represents a first improvement of the classification criteria structure of the catalogue, increasing the number of criteria applied to the assets from 54 to 62 . Multi-criteria approaches, such as the one developed by the authors, are considered of great interest, and it is actually possible to identify other proposals for the study of cultural heritage based on these techniques [20-22]. Thus, not only the previously obtained trends are reviewed, but also new possible analyses are exposed, and also in achieving this goal, the geolocation information had a key role, as will be exposed below.

Thus, this work proposes several analyses of the industrial heritage in the Autonomous Community of Andalusia, all of them by using the developed catalogue. These analyses enable characterizing this heritage typology in this territory and the review trends that were previously obtained, but they also show the potential of the catalogue as a tool for the study, promotion, and management of these assets. Furthermore, the incorporation of new classification criteria represents the first enlargement of the criteria structure of the catalogue. This means an increase in the variety of analyses and approaches enabled by this tool, and the improvement of the catalogue as the response to a need identified during its use as support for research activity [19]. In that sense, new possible lines of improvement are commented upon, and the main conclusions of the work are finally exposed.

\section{Methodology}

In this section, the main methodological aspects that were applied in this work are exposed. Thus, the growth process of the set of assets, the criteria structure of the catalogue, its use as analysis tool, and the special considerations of some of the classification criteria considered are defined and explained.

\subsection{Set of Assets Increase}

The first task addressed in this work was the identification and selection of new assets of interest in the territory considered as study case, which is in this case Andalusia. This searching work was not only an additive process focused on the increase of the set of assets considered, but also a review of that set and its capability of being representative of this typology in Andalusia. Perhaps the first goal that comes to mind when a recording initiative such as this one starts is achieving a wide enough set of elements to be representative of the reality that is to be characterized. In the case of the industrial heritage, the large number of assets falling under this term makes the identification of all of the elements difficult.

Nevertheless, the catalogue developed by the authors is not only a list of assets of interest, but also a whole criteria structure that is applied to all of them, so one of the main opportunities that the catalogue offers is the wide variety of analyses allowed by combination of these criteria. While accepting that including all of the existing assets of this type is very difficult, the wider the set of assets is that are considered, the more reliable and representative the trends of the territory that are observed through the run analyses. In this sense, the number of assets that is included in the catalogue for Andalusia was increased from 166 to 350, which means more than double the entries that were initially considered for this territory.

Figure 2 shows in blue the initial distribution of assets through the eight provinces of the Autonomous Community of Andalusia. The new assets that are included for each territory are marked in orange, and the total number of assets is indicated above each bar in the graph. 


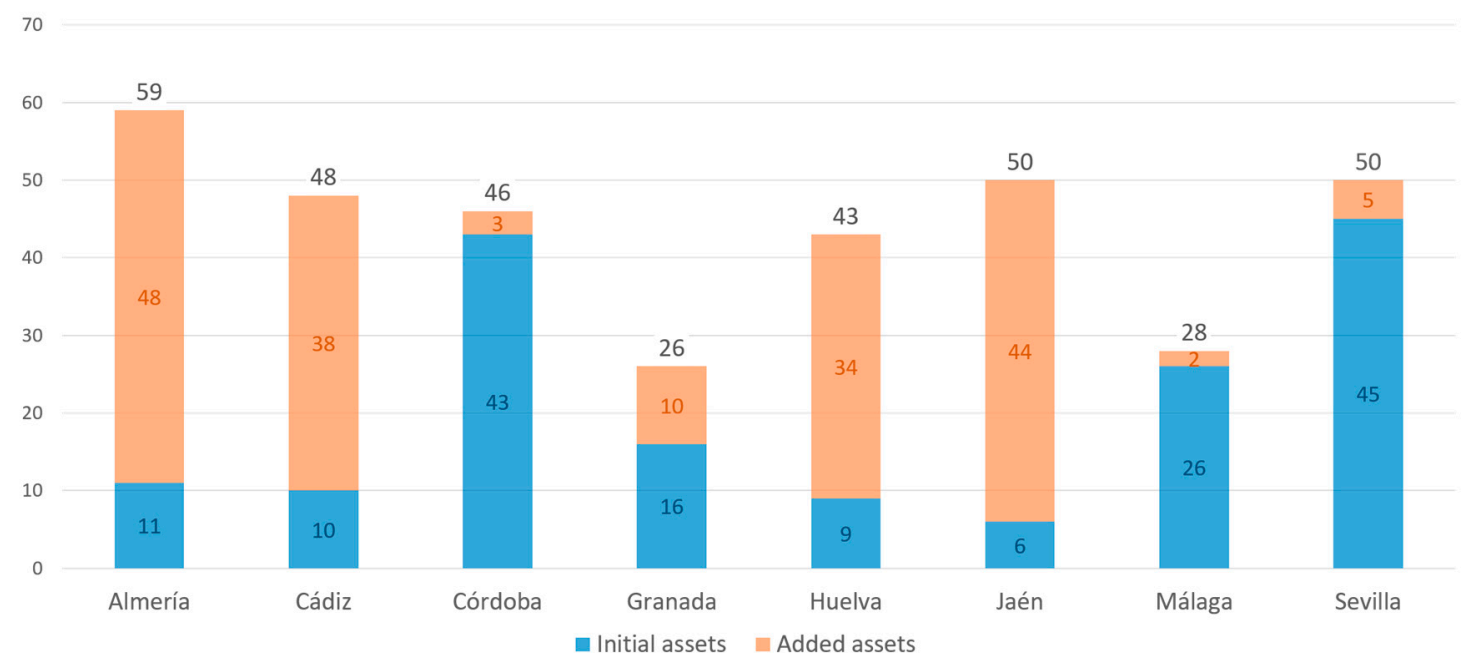

Figure 2. Assets initially considered for each province: new assets included, and actual number of assets identified.

Firstly, it is possible to appreciate significant changes from the initial distribution. Thus, territories with less importance at the initial distribution, such as Almería, Cádiz, Huelva, and Jaén, now have a greater role. On the other hand, territories with the highest number of assets at the initial distribution-Córdoba and Sevilla-and the ones in intermediate positions-Granada and Málaga-have experimented much lower increases. This can be perceived as an attempt to equilibrate the distribution trend, but similar resources and efforts were inverted in all of the cases. The new territorial distribution after the actualization of the catalogue will be analyzed in more depth in the later sections.

Another consequence of this study focused on Andalusia is the imbalances that the actualization of the sample in this territory causes in the territorial distribution at the national level. Before this work, Andalusia was the third territory by the number of assets included in the catalogue, only behind Catalonia and Madrid, and very close to the Basque Country. Actually, its 350 assets make it the leading territory in assets contribution to the catalogue. Due to this, similar works must be developed for each one of the other autonomous communities, and for those future works, the present study must establish a reference baseline.

\subsection{Criteria Structure}

In this section, the classification criteria structure of the catalogue is briefly exposed, differentiating between initial and new criteria, and showing the logic and the functioning of the catalogue. However, after the general overview of the criteria structure, some guidelines for the application of some classification criteria are commented. Despite all of the criteria considered being attributes that are easy to understand, their application is not always so simple. Classifying any kind of elements in relation to a particular aspect and under a finite number of categories can cause problems. When such a wide set of elements must be processed, there will be particular situations in which the boundaries between the classification categories can be confusing. Having some simple selection guidelines will be very useful in those cases.

Figure 3 shows the criteria structure of the catalogue. As shown in the figure, the criteria that are considered can be grouped along three general categories. The first one includes information that is able to define each asset from different approaches. Thus, the proposed designation for the asset, information concerning the location of the asset, the definition of the scale of the asset, the technological level of the asset, the role in the productive process, the corresponding industry sector, and an interactive link to a website with content related to each particular asset are included in this first category of the catalogue. A second group of criteria is focused on the presence or not of each 
asset into cataloguing initiatives of special interest, as well as the protection, when it exists, as a Good of Cultural Interest (BIC). Finally, a third group of asset enables identifying those assets that are reused for new activities, and for those cases, the nature of these activities also.

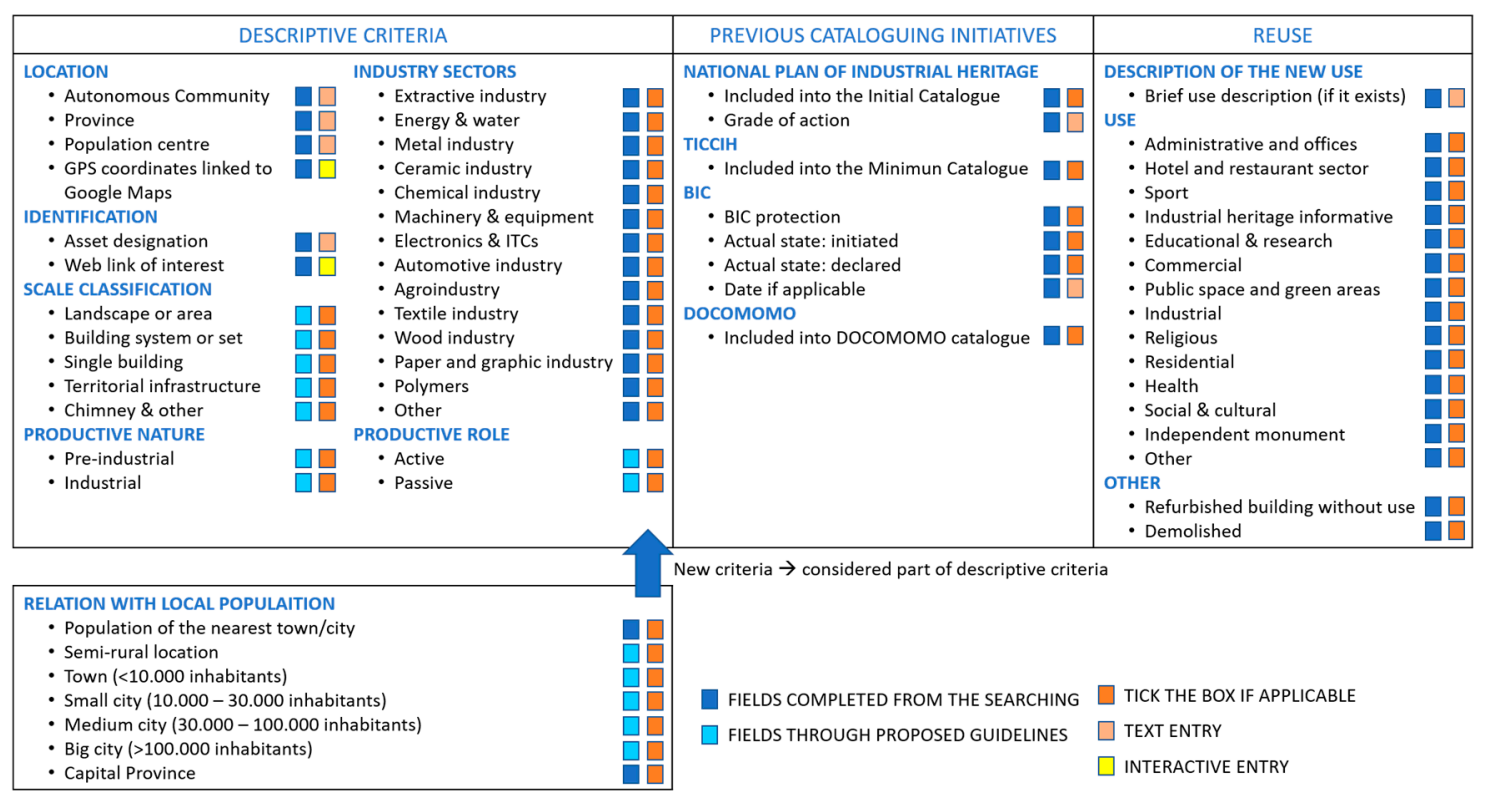

Figure 3. Criteria structure of the developed catalogue: information obtainment process and type of information.

Figure 3 also indicates the nature of the information contained in each field. First, with regard to the information obtainment process, it is necessary to differentiate between those fields completed through only information searching work and those which required some interpretation guidelines. Secondly, the way in which the information of the different fields is shown in the catalogue is also indicated. The most common procedure is a simple mark in the corresponding field when that characteristic is noticeable in the analyzed asset. In other cases, text entries are necessary in order to describe the information of that field. In addition, two fields with interactive information are included for each asset. One of them is linked to online information of interest for each single asset, and the other one contains the global positioning satellite (GPS) coordinates of the corresponding asset, and is linked to Google Maps.

New Criteria Added to the Structure of the Catalogue

In addition to the criteria considered in the initial version of the catalogue, new criteria were incorporated to the structure. This new set of features can be understood as new descriptive criteria, since they contribute to the characterization of the analyzed assets. Figure 3 suggests this idea. The aim of these new criteria is to determine the relation of the industrial assets considered with the existing local population. This relation, and the potential synergies issuing from it, are of great interest when the possible reuse of one asset is being discussed. In fact, as previously mentioned, the interest of including this group of criteria was detected through the use of the catalogue as part of a global methodology, in which the information contained in the catalogue supports multi-criteria decision structures for the selection of the most appropriate new uses for these kinds of goods [19]. As in the case of the previously exposed criteria groups, the nature of the information contained in each field is also indicated in Figure 3.

Table 1 illustrates how the eight criteria added to the structure of the catalogue try to define the relation of the industrial assets that were analyzed with the local population. Thus, two implicit classification levels can be identified through these eight criteria. First, they allow differentiating 
between rural and urban environments. Then, inside each of the two main categories, new particular situations are considered. For rural environments, the relationship with the local population is then analyzed. In the case of assets located in urban environments, the size of the population center is indicated. Special aspects are also considered, such as the special role that the province capitals have in each region, and the number of inhabitants of the nearest population center, according to the data provided by the National Statistical Institute of Spain [23].

Table 1. Approach of the new classification criteria added to the catalogue structure.

\begin{tabular}{|c|c|c|}
\hline First Level of Classification & Second Level of Classification & Catalogue Denomination \\
\hline Rural environment & $\begin{array}{l}\text { Relation or not to any human } \\
\text { settlement }\end{array}$ & $\begin{array}{c}\text { Rural location } \\
\text { Semi-rural location } \\
\text { Town }\end{array}$ \\
\hline Urban environment & City size & $\begin{array}{l}\text { Small city } \\
\text { Medium city } \\
\text { Big city }\end{array}$ \\
\hline Complementary information & $\begin{array}{c}\text { Special role } \\
\text { Population measurement }\end{array}$ & $\begin{array}{l}\text { Province capital } \\
\text { Population of the nearest town }\end{array}$ \\
\hline
\end{tabular}

\subsection{Application Guidelines}

As exposed before and indicated in Figure 3, some classification criteria need basic guidelines when they are applied to the set of assets of the catalogue. In this section, some orientations are proposed in order to make the classification through these criteria easier and clearer.

\subsubsection{Scale}

One of the most interesting characteristics of the industrial heritage, as the typology of the cultural heritage, is the variety of scales in which these assets can appear. The criteria structure of the catalogue includes five different categories for the classification by scale: chimneys and other, immovable asset, system or building set, landscape or area, and territorial infrastructure. All of these categories are easy to understand through examples, but also it is possible to give many examples in which the boundaries between categories are not so obvious. In order to help with classifying each good by this criterion, the authors defined a set of aspects that can be perceived in a different way from the perspective of each classification category [1]. Figure 4 resumes the initial guidelines.

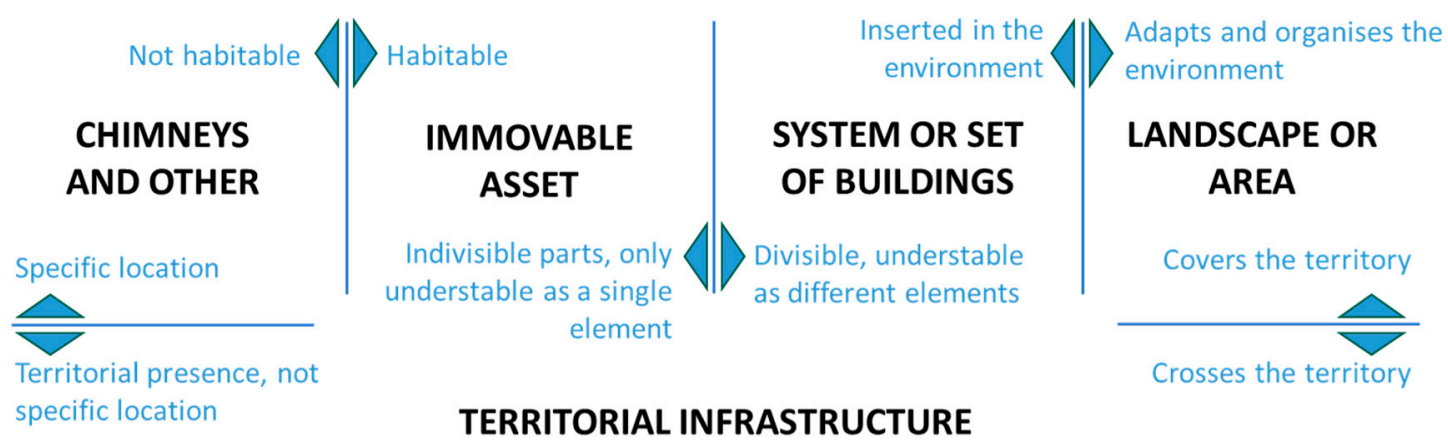

Figure 4. Graphical guidelines for the classification through the scale criterion.

The category "chimney and other" is used when only the remains of some isolated parts form the original state of the asset. The most usual example of this situation is industrial chimneys, but other elements such as melting furnaces are also included in this group. These kinds of elements are not movable assets, but they are not immovable assets, either. Nevertheless, some of these elements can be really large, as in the case of blast furnaces, so they can even perceived as buildings by many people. Figure 4 identifies the habitability as the key aspect to be considered when the studied good did not 
belong clearly to either of these two categories. Chimneys, furnaces, and other similar elements have been built using the same constructive systems used in the industrial buildings in which they were included, such as for example using bricks, and they can be big, too; so, effectively they can be really close to the building scale. However, they were not designed to be inhabited, and there are not real living spaces in them.

The limits between the category of "immovable asset" and "system" or "set of building" will be confusing. It is easy to distinguish between a single building and a set of them. However, there are buildings that are the result of the sum of different spaces that define various independent volumes. In these cases, it seems as if various different buildings had been joined into only one. This idea can be exposed clearer in the opposite sense. It is usual that industrial facilities, with a long enough period of activity, experiment with changes due to the adaptation of the installations to new needs. One often action in that context consists in the covering of the space between two industrial buildings in order to expand the facilities. However, this does not mean that those two separate buildings are only one, even though they can be seen in that way. When classifying an asset in that situation, its analysis will not be focused only in the constructive appearance as a single building, and its more complex nature will be detected.

Thus, the same approach can be applied to other similar situations. There are industrial buildings in which it is possible to identify enough independent elements. However, the application of this idea can be confusing, so a clear guideline must be established. An approach to the problem through reuse actions in large industrial facilities can provide the key aspect to be considered. When this type of asset is recovered for new uses, usually the whole facility is not reused, but some elements are, even for different activities, and this is a demonstration of their independence. Thus, when analyzing great buildings, which also could be understood as the result of a sum of different volumes and productive spaces, thinking about the real possibility of acting on only some of those above parts is what can help determine whether enough independence exists between them for them to be considered as different elements, or not. Figure 5 illustrates this idea graphically: on the left and right are representations for the concepts of a single asset and a set of assets, and in the middle area of the figure, intermediate situations are shown. Thus, the capability or not of acting only on particular parts is what determinates the classification as a single asset or as a set of assets.

\subsubsection{Technological Level}

Two classification fields are included regarding to this aspect. Thus, assets can be identified as "pre-industrial" or "industrial". This classification can seem easy, but when you classify an element you are applying some criteria, and sometimes the decision about which are the appropriate criteria can be complicated and the subsequent application of the selected criteria can also be confusing.

The definition of industrial heritage that was proposed by the National Plan of Industrial Heritage of Spain in 2001 [2] identified the assets that compound this typology as the one result of the relation between a social model, capitalism, and the technological resources of the machining, which constrains these assets within the period between the second half of the 18th century and the last third of the 20th century. This temporal limitation is similar to the one proposed by The International Committee for the Conservation of the Industrial Heritage (TICCIH), considering the same start and an as of yet undefined end that goes on until today. 


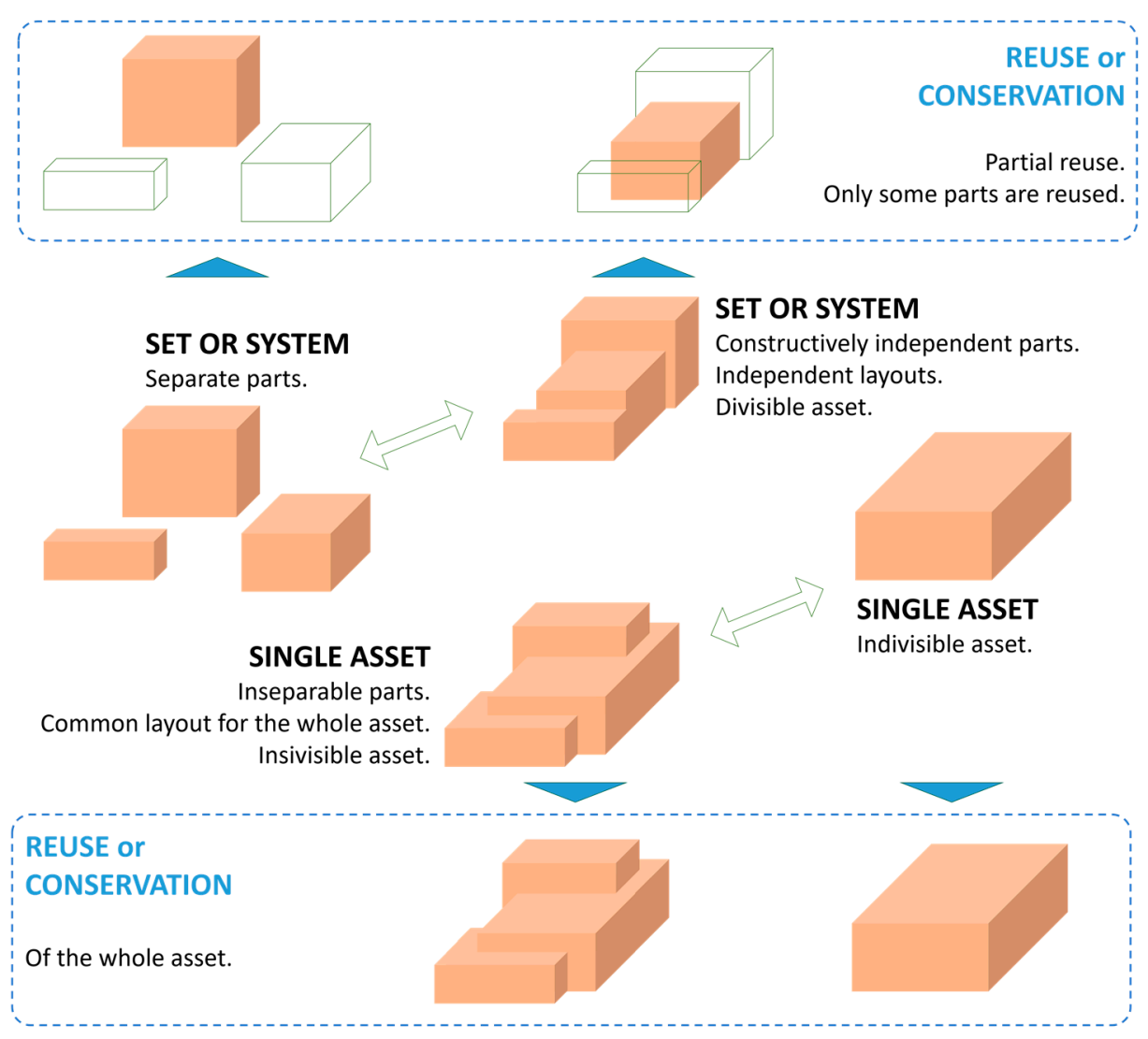

Figure 5. Graphical guidelines for the classification as a single asset or as a set of assets.

These general approaches define a limit between an industrial era and a pre-industrial era, and that idea was discarded in this work. The authors understand that the concept of industrial heritage must be able to cover and include any element-both movable and immovable assets—-that are capable of helping the understanding of the operation of productive processes of interest, and also the life around these activities. The corresponding historical period is important information and in this study it is used as a classification criterion, but it is not a reason for the exclusion of a group of assets of interest, nor for the preference of some of them.

Thus, after the decision includes both possibilities in the catalogue, the guidelines for making the decision in each particular case must be established. The limits between industrial and pre-industrial assets might sound simple, but in many cases, it will not be so clear to decide in which group a particular asset must be included. The operation period of the asset is not a key aspect in this respect. Pre-industrial assets such as windmills were operating (and also were built) during the period defined by the National Plan and TICCIH as industrial. So, what criteria could be of help?

For that purpose, the authors established two main aspects that must be analyzed before the classification of each particular asset as pre-industrial or industrial [1]. On the one hand, there is the technological level of the asset, but this level is from a particular point of view that is focused on the power source that was employed. When this source is linked to the natural resources of the environment, the location of the asset is conditioned by the need of proximity to that resource, and this is understood as a pre-industrial attribute, in contrast to industrial situations in which steam engine or electric energy are the usual power sources. However, special situations can exist, so a second aspect is analyzed: the productive level or capacity. When the productive activity supplies the area near the asset, the productive level is understood as local, which means a pre-industrial attribute for the classification into the catalogue. When the asset is focused on exporting the products to other areas or regions, the productive volume is higher, and this is understood as an industrial attribute. 
By combining the conclusions of both analyses, it is possible to select the industrial or pre-industrial nature of each particular asset. There will be situations in which these conclusions will point in opposite directions, and then the trend that is valued as the one of higher relevance will be one considered for the final classification of the asset.

\subsubsection{Relation with Local Population}

As exposed before, one of the main functions of the developed catalogue is the support of other initiatives of study, promotion, or actuation. In that sense, the catalogue was initially integrated in a global methodology for the study and management of the industrial immovable assets that have been developed by some of the authors, concretely for the selection of the most compatible new uses for particular assets, in order to minimize as much as possible the impact of the adaptation to the new activity [19]. Precisely in that context, the interest of including some new criteria in the catalogue was identified. When one of these assets is recovered for a new activity, one of the most important aspects is the success of this activity. When the new use fails, two new scenarios emerge for the asset. On the one hand it can be again in a situation of abandonment for some time, which probably will deteriorate the asset in some way. On the other hand, it can be adapted again for a different new use, and that process will have some kind of new impact. Both situations are undesired, so making the right selection of the new use is a key aspect.

In that sense, the criteria structures of the decisions of the mentioned global methodology demand the inclusion of new criteria into the developed catalogue. The environment of the assets is very important for the success of the potential new uses. For example, some uses will benefit from highly populated environments, while other activities can be more appropriate for rural contexts. Thus, in addition to the location criteria that are initially included into the catalogue, criteria related to the rural or urban context of each asset considered, and the size of the surrounding villages and cities have been included in the catalogue. Thus, eight new categories were considered and included into the catalogue as new classification criteria. These new criteria were listed below in Table 1, which also shows the logic behind the new criteria incorporated into the catalogue, but not the needed application guidelines.

In the case of rural environments, two possible situations are considered. The classification as a "rural location" is used when the analyzed asset has no direct relation to any nearby village or town, either because a nearby village or town does not exist, or because there is not a clear connection with the analyzed asset, for example a road. Thus, this criterion of classification shows a certain grade of territorial isolation. The criterion called "semi-rural location" can represent assets out of the urban fabric, but with certain connection and relation to some nearby town or village, for example due to its close proximity or for the existence of a road, and also it can be used for assets associated to small groups of houses without their own real administrative or fabric structure. As it is possible to appreciate, assets classified with this criterion will be neither included in large urban structures nor connected to them, but they will not have the previous level of territorial isolation. If potential synergies with human settlements can be identified, then the potential demand of certain new uses can be taken into account.

On the other hand, four categories are defined for assets located in urban environments. The category called "town" is used for populations of less than 10,000 inhabitants. When the number of inhabitants is higher than 10,000 the term city is used, while "small city" is when the population is between 10,000-30,000 inhabitants, "medium city" is when the population is between 30,000-100,000 inhabitants, and "big city" is when the population is higher than 100,000 inhabitants. Figure 6 shows graphically the key aspects for decision making about the most appropriate category of classification in each case when these new sets of criteria are applied to each particular asset. 


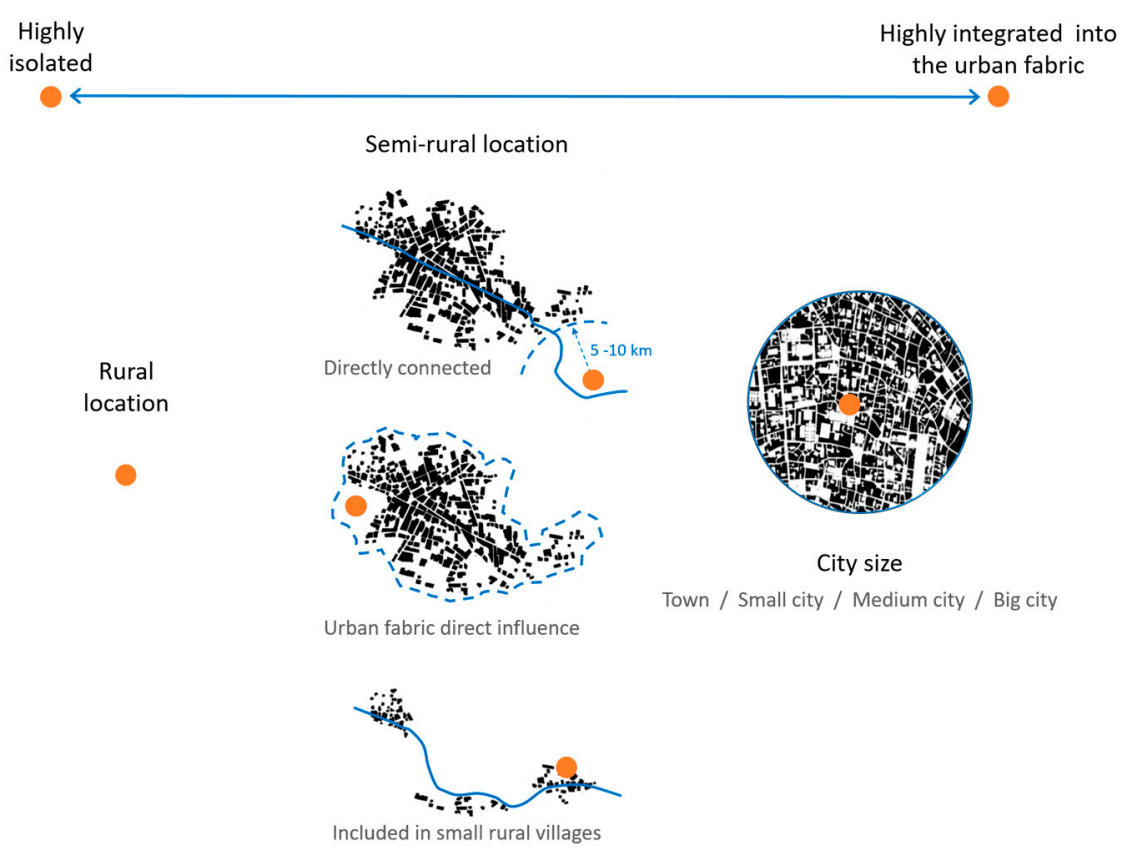

Figure 6. Guidelines for assets classification by criteria related to the grade of relation with human settlements.

In addition, the special role as "province capital" is also considered. When the population intervals were defined, the number of inhabitants of the main cities and towns of Andalusia was consulted in the database of the National Institute of Statistical of Spain [23]. Table 2 shows that, together with the eight province capitals, other cities have an important population in Andalusia, cities in which different industries are present, and contribute with some elements to the catalogue, so this additional classification criterion was considered of interest.

Table 2. Cities with the highest population in Andalusia. Source: National Institute of Statistical of Spain [23].

\begin{tabular}{cccc}
\hline & \multicolumn{3}{c}{ Population } \\
& $\mathbf{2 0 1 5}$ & $\mathbf{2 0 1 6}$ & $\mathbf{2 0 1 7}$ \\
\hline Sevilla & 693,878 & 690,566 & 689,434 \\
Málaga & 569,130 & 569,009 & 569,002 \\
Córdoba & 327,362 & 326,609 & 325,916 \\
Granada & 235,800 & 234,758 & 232,770 \\
Jeréz de la Frontera (Cádiz) & 212,876 & 212,830 & 212,915 \\
Almería & 194,203 & 194,515 & 195,389 \\
Huelva & 146,318 & 145,468 & 145,115 \\
Marbella (Málaga) & 139,537 & 140,744 & 141,172 \\
Dos Hermanas (Sevilla) & 131,317 & 131,855 & 132,551 \\
Algeciras (Cádiz) & 118,920 & 120,601 & 121,133 \\
Cádiz & 120,468 & 118,919 & 118,048 \\
Jaén & 115,395 & 114,658 & 114,238 \\
\hline
\end{tabular}

The application of this group of criteria, as well as the other ones included in the catalogue, is supported by the geolocation information included for every asset of the catalogue. This aspect is exposed in the following section.

\subsubsection{Geolocation Information as Support for Cataloguing Criteria}

Geolocation information represents a data field with special potential from many different points of view. As mentioned before, for each asset included in the catalogue, the GPS coordinates are shown, 
and when selecting this field for a particular asset, the location of that element is shown in Google Maps. Having every asset of the catalogue linked to Google Maps [23] has an enormous potential and offers many opportunities, both as a promotion tool for this typology and as support information when particular assets must be classified through some of the criteria considered in the catalogue.

Figure 7 shows some of the main possibilities that this data field offers. Having this information for all of the assets of the catalogue, it is possible to make maps of assets by using Google My Maps. In these maps, it is possible to include a set of assets that has specific characteristics, such as the location in a particular region, the activity sector, or any other aspect included in the catalogue as one of the classification criteria. In addition, for each single asset, it is possible to make a virtual approximation or visit through the functions included in Google Maps [24] such as aerial views, Street View, and the photographs loaded by users.

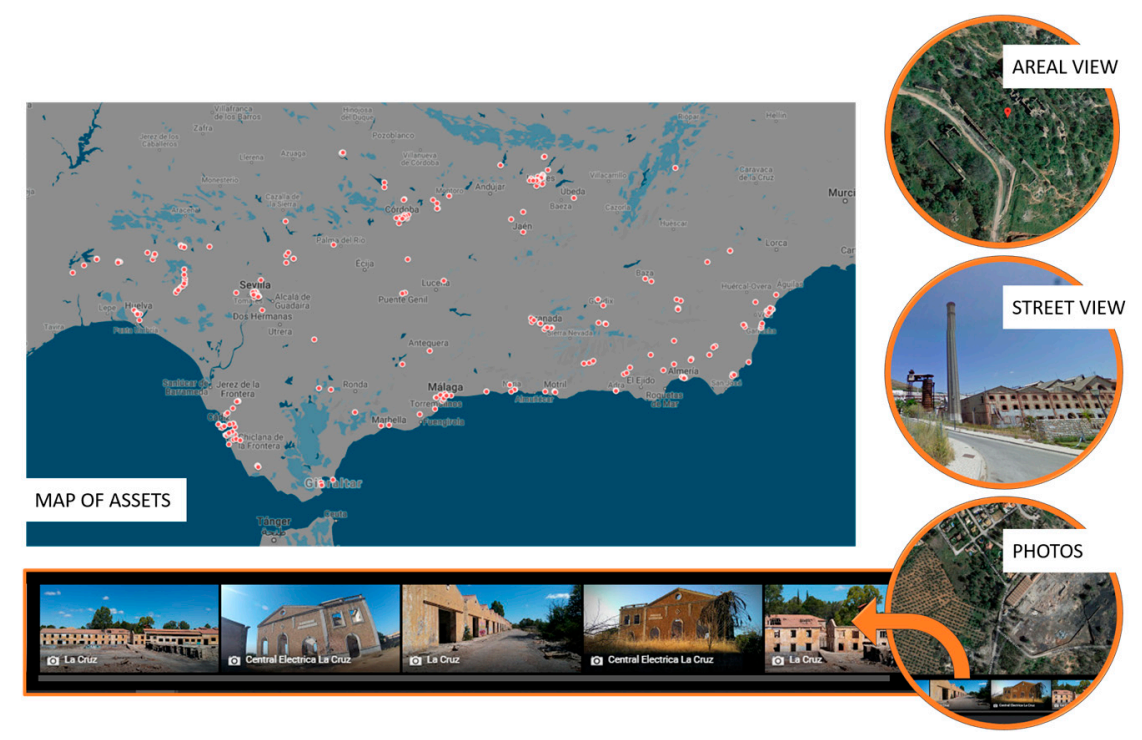

Figure 7. Functions included in Google Maps.

Thus, this resource has significant potential applications as a tool for the diffusion and promotion of these assets, as well as for their visitors, so the interest in the field of tourism is considered relevant. However, in addition, during the application of the classification criteria considered in the catalogue the geolocation information also has a key role. In many situations this information will help to apply properly some classification criteria, mainly the scale criterion and the relation with local population. In both cases the geolocation information and the different virtual view modes allowed by it, make possible to evaluate and select the most appropriate category for each analyzed asset.

\section{Results and Discussion}

Figure 8 allows a combined interpretation of the distribution of assets, both by their location into the different eight provinces of Andalusia, and by the sector of activity. Thus, a vertical reading of the graph provides an interpretation of the distribution of assets for each sector of activity through the eight provinces. When the graph is read horizontally, the distribution of assets into the different sectors can be observed for each territory. In addition, at the top of the graph and on the right side, the general distributions of the total number of assets that have been identified for each territory and for each activity sector are shown. Thus, regions and activities with special relevance and presence within the set of assets that are being considered can be identified, and then distribution trends can be observed through vertical and horizontal interpretations of the graph. 


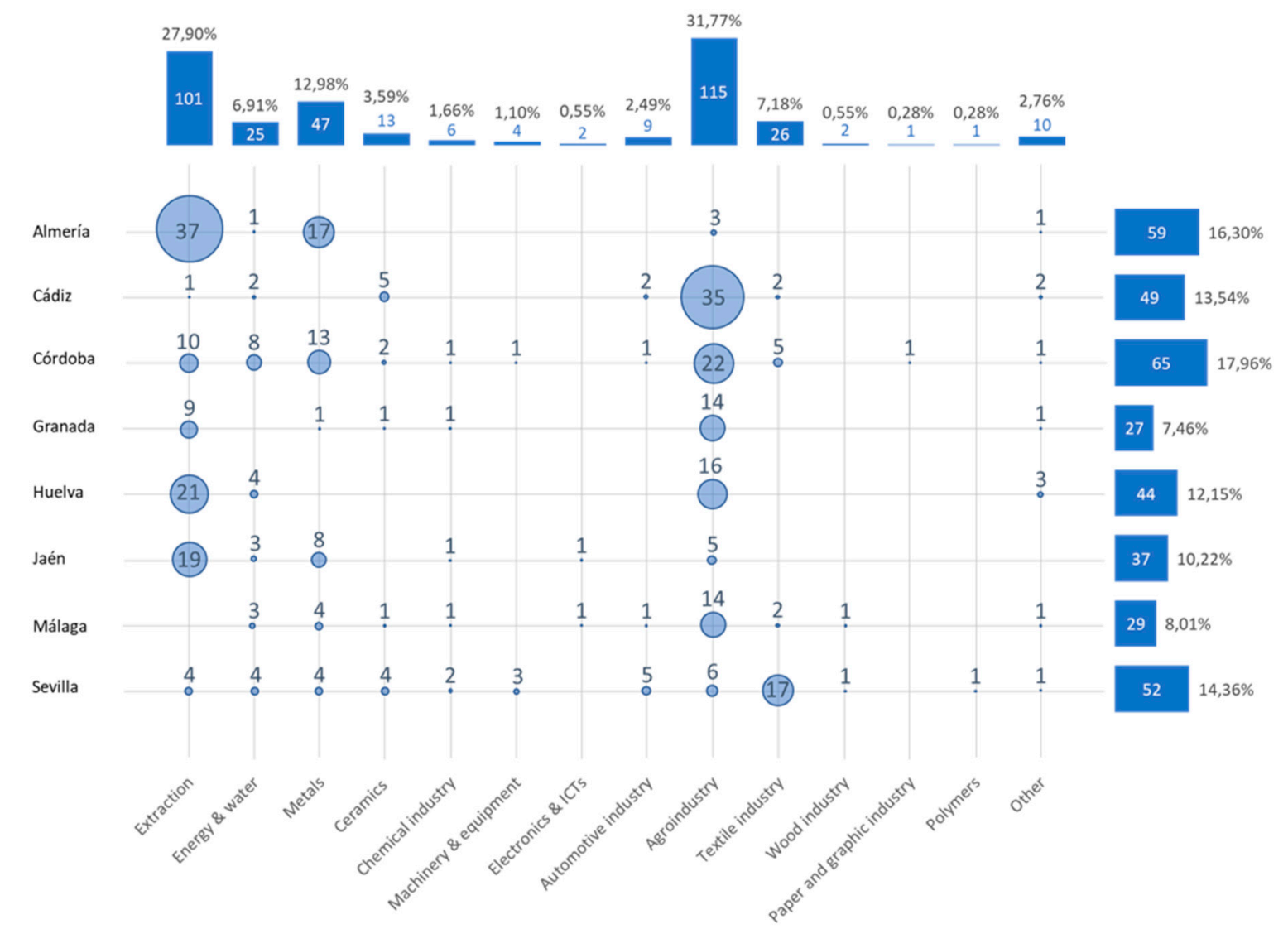

Figure 8. Distribution of assets simultaneously considering location and sector criteria.

At the same time, the different scales of the nodes in the graph enable identifying situations of special relevance. Examples of this are the high presence of assets linked to extraction activities in Almería and agroalimentary industries in Cádiz. The identification of these high presences is of high interest, and will derive in deeper studies in order to appreciate the circumstances behind these trends. Thus, in the case of Cádiz, by considering the scale criteria, it is possible to appreciate the significant presence of an immovable asset scale. When the set of assets is consulted, it is possible to identify a great number of windmills, which are the elements that make the presence of this activity sector so important within the distribution trends that are analyzed. Figure 9 shows this situation graphically. In addition, photographs of some examples of the identified windmills are included.

From a different point of view, the reutilization of this kind of assets for new activities is another aspect that is of the highest importance. Understanding the developed catalogue as a support tool for hierarchical multi-criteria structures, the identification and then the analysis of the reutilized assets represent key information.

In Figure 10, using a graph similar to the one used in Figure 8, the distribution of assets that simultaneously considers the criteria related to the location and the new uses is analyzed. At the top of the graph, the distribution of assets that is reutilized for each possible new use is shown, considering the assets located in every province. Thus, this trend represents the global distribution for this approach for the Autonomous Community of Andalusia. This analysis is of high interest, so it enables identifying the uses that have been able to activate new possibilities for assets that had lost their original function, and which needed a new one for their sustainable conservation. Consolidated examples of assets reused for new activities represent information of great value in order to establish appropriate strategies for the reutilization of this type of assets, providing examples of both good and bad practices. 


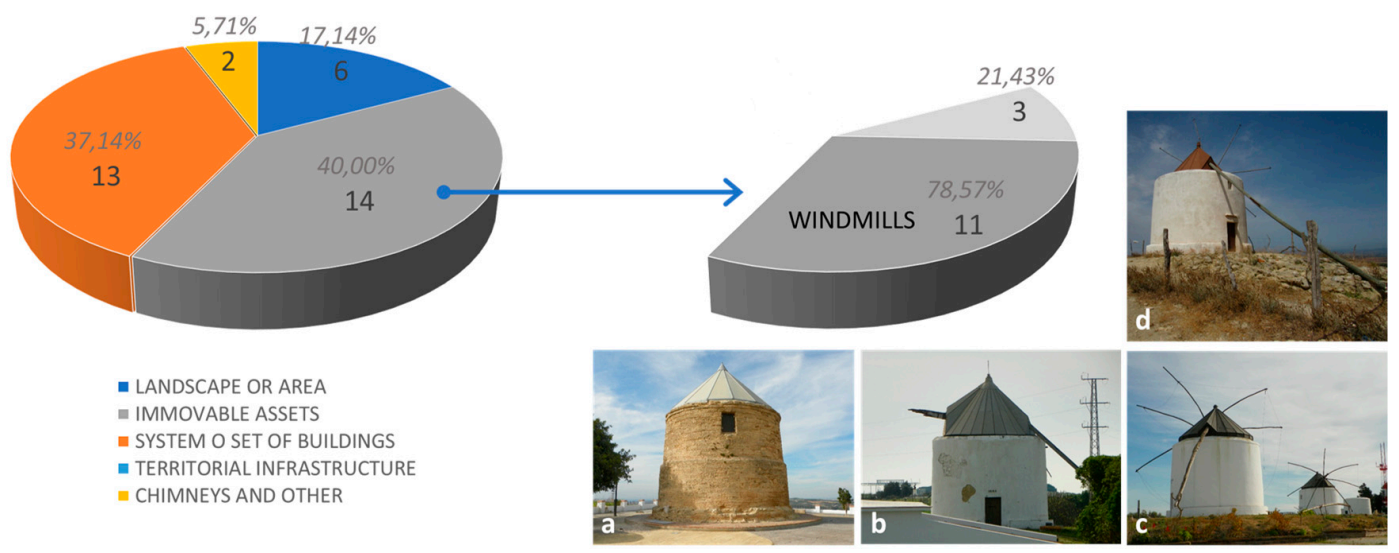

Figure 9. Distribution of assets for the scale criterion applied to the agroindustry assets identified in the Province of Cádiz. The presence of windmills and some examples in Vejer de la Frontera: (a) and (b) windmills at "Loma de la Buenavista"; (c) windmills that neighbor San José; (d) windmill "la Cruz de Conil".

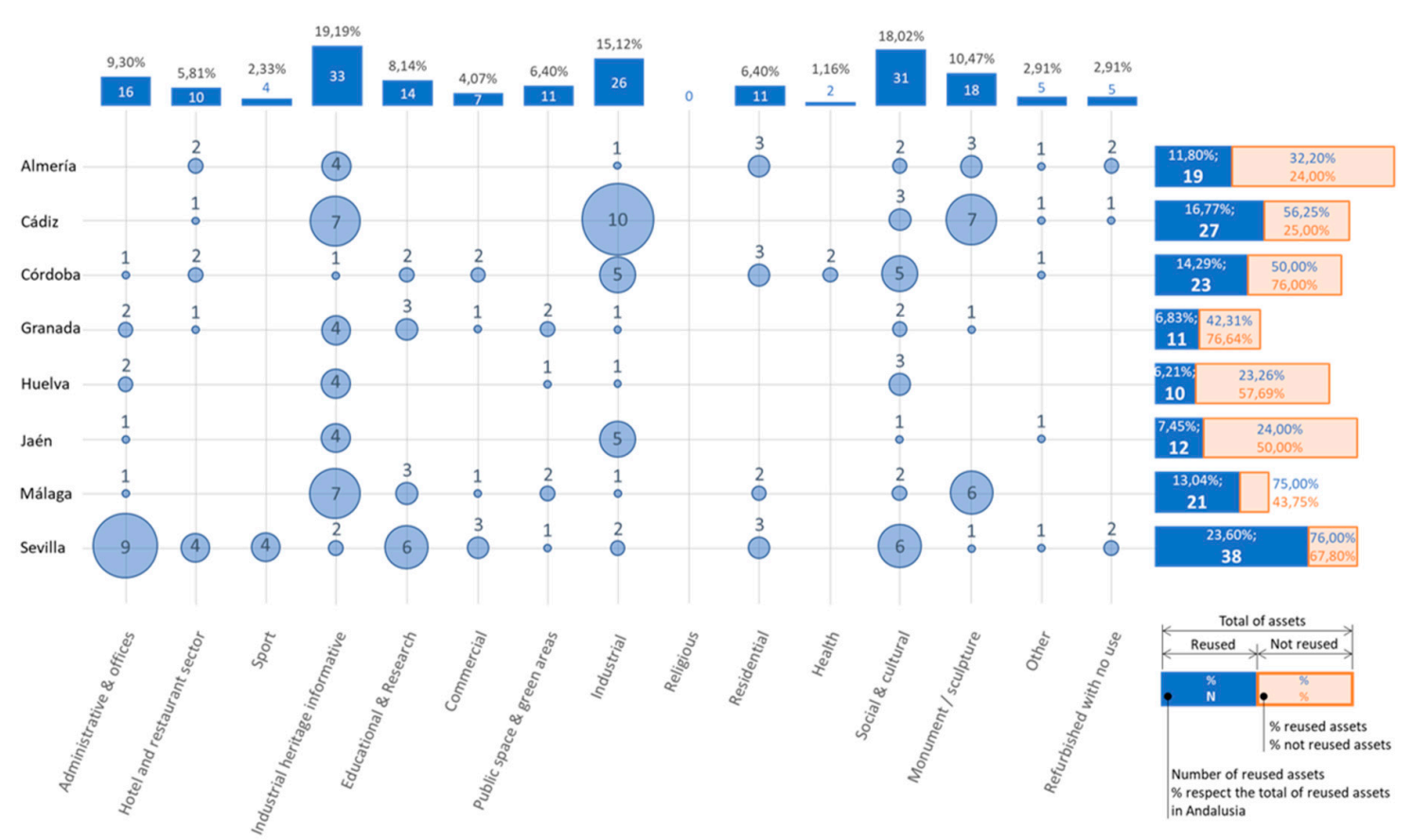

Figure 10. Distribution of assets simultaneously considering location and the new use when it exists.

In addition, it is possible to make a similar horizontal interpretation of the graph for each province. This enables appreciating the particular trends within each territory and identifying the most relevant new uses in each case.

When Figure 10 is interpreted vertically, it is possible to appreciate the presence of the assets identified for each new use within every particular territory. This enables identifying the special relevance of some sectors of activity in particular territories. For example, in the case of the use identified as "administrative and office", the graph shows the especially significant importance of the Province of Sevilla with regard to this use. At the same time, for the uses with highest presence, which are the ones identified as "industrial heritage informative" and "social and cultural", the graph shows trends with more balanced presence distributions in each territory, but it is also possible to identify the most significant provinces in each case.

On the right side of the graph, the total reutilization of assets observed in each territory is shown. This global analysis does not take into account the specific nature of the new activities, but rather 
considers the total number of assets reused through the different new uses in each province. Both the number of assets reused in each territory and the percentage of them within the total number of reused assets in Andalusia are indicated. Furthermore, the amount of reused assets in each territory is related to the total number of assets considered in the catalogue for the corresponding province. Thus, for each territory, the blue bar corresponds to the reused assets, and the orange bar corresponds to the ones that have not been recovered for new activities. Within each orange bar in the graph, the percentage of reused and not reused assets for each territory is indicated, using blue and orange text respectively in order to add clarity.

Furthermore, as in the graph shown in Figure 8, the size of the nodes makes a fast and easy identification of special and relevant situations possible, such as the previously mentioned presence of the use that is denominated as "administrative and offices" in the Province of Sevilla, or the significant number of assets dedicated to industrial activities in the Province of Cádiz. The identification of these and other high presence situations within the graph enable proposing and defining new analyses when necessary.

Since information about the reutilization of these assets for new activities is important, both as a sustainable strategy for their conservation and as support for the multi-criteria decision structures included in the global methodology developed by the authors [1,19], further analyses are exposed in order to show the potential of the catalogue as a useful and flexible tool.

Figures 11 and 12 propose new approaches by considering simultaneously three different categories of criteria: location, new uses, and scale. Figure 10 identifies "industrial informative heritage" and "social and cultural" as the uses with higher number of assets. On a second level, it is possible to identify three uses: "administrative and offices", "educational and research", and "monument/sculpture". These uses of special presence represent the most consolidated reuse strategies of this typology in Andalusia, and analyzing the possible influence of characteristics such as the scale of the reused assets represents a very interesting approach. Thus, Figures 11 and 12 incorporate a new criterion into the analysis shown in Figure 10, the scale of the corresponding assets, in order to identify possible relations between the activities established and the scale of the affected assets.

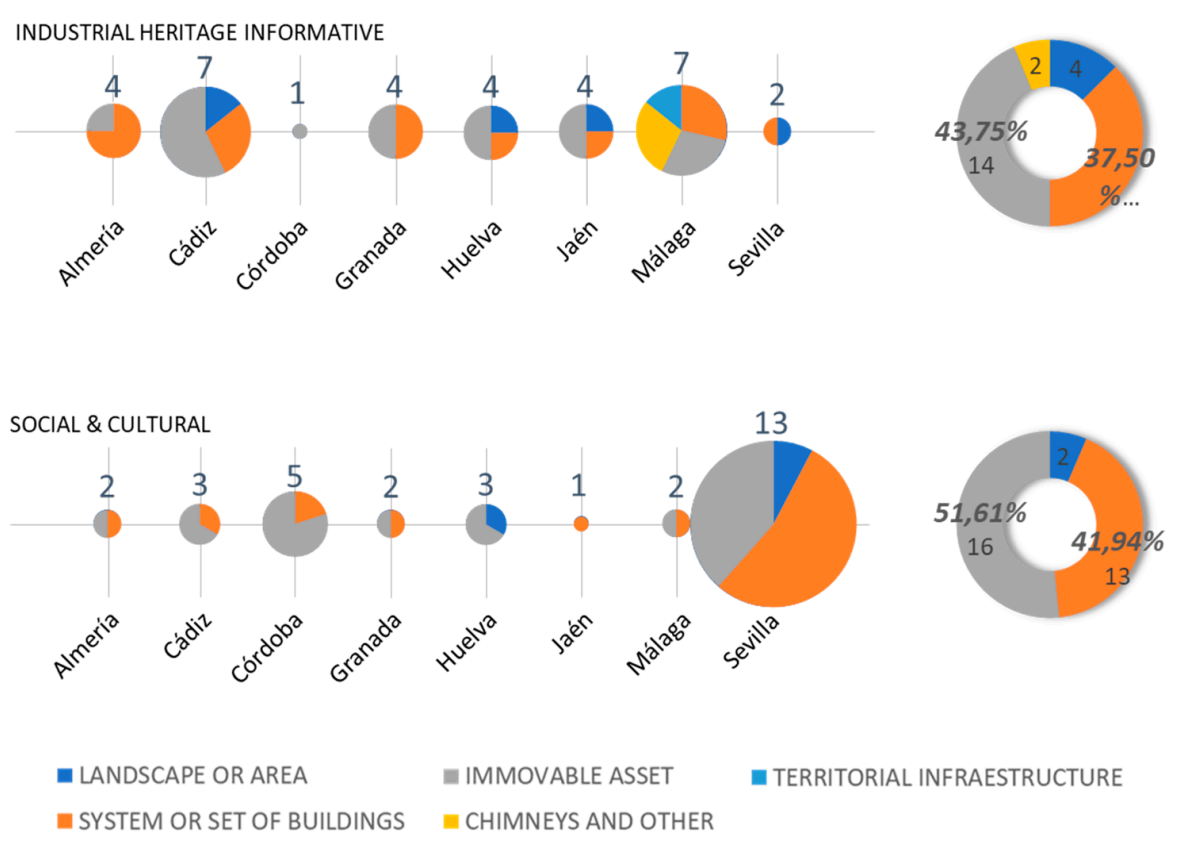

Figure 11. Distribution of assets within the two new uses with the highest number of assets and also considering the location and the scale criteria. 

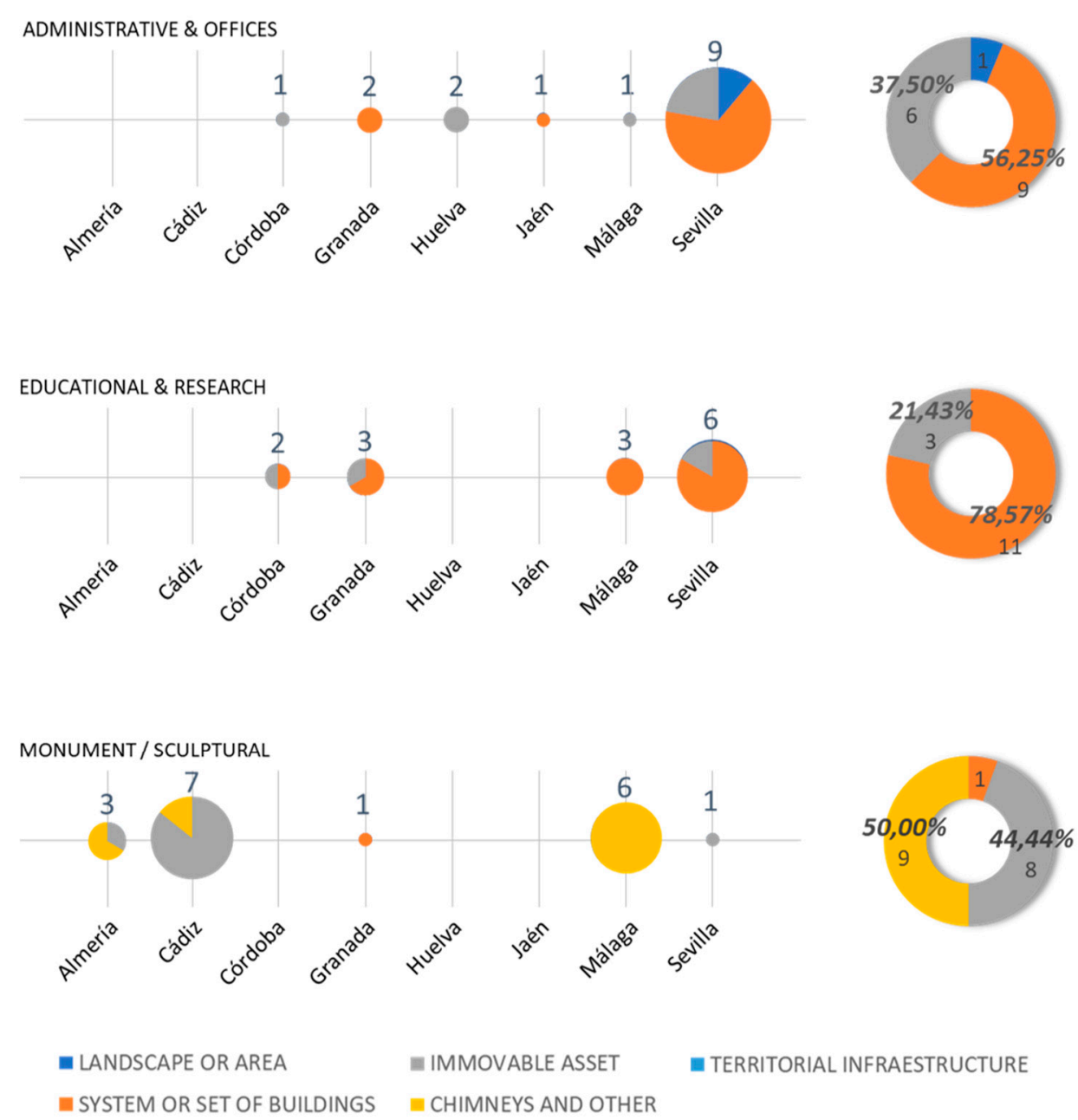

Figure 12. Distribution of assets within the new uses with a significant number of assets and considering the location and the scale criteria.

At the top of Figure 11, the distribution of assets through the eight provinces of Andalusia and the presence in each case of the scale categories considered into the catalogue are shown for the use "industrial heritage informative". At the bottom of Figure 11, the same approach is applied to the assets corresponding to the use "social and cultural". In both cases, a representation of the total distribution of scales for the analyzed use is shown on the right side of the figure. The same approach is applied in Figure 12 to the other three analyzed uses.

Observing both figures, it is possible to appreciate some trends of interest. Firstly, it can be noticed that the "territorial infrastructure" scale does not have presence. This highlights the greater difficulties that the assets of this scale present in terms of reuse for new activities. Regarding the use of "industrial heritage information", the other four scale criteria that are considered in the catalogue have representation, and in most of the cases, in several different territories. This heterogeneity is due to the nature and the objectives of this possible use, whose main purpose is not only the conservation, but also the dissemination and promotion of the concerned asset and its heritage values. Thus, it is not so much the scale demanded by the new use, but the scale of the original asset itself that persists, since the scale itself is a distinctive feature, and a characteristic of the value that must be preserved and shown. In the other cases, it is possible to identify a clear prevalence of some scales over others.

In the case of the use named "social and cultural", two scales have a clear predominance without signs of a significant preference for either one of them. Thus, both the "immovable asset" and the "system or set of buildings" scales emerge as the main alternatives for this use. In addition, 
two examples of "landscape or area" scale appear. This is due to isolated situations derived from the classification guidelines established for the construction of the catalogue [1]. Industrial landscapes and areas represent dimensions clearly different from other scales such as industrial facilities composed by a set of buildings, so this kind of elements must be identified as such. However, other criteria of the classification structure applied into the catalogue may affect only some of the recognizable single elements that are included within that landscape or area. The reuse of some of the elements while the others are abandoned, or the legal protection only of some of them, are examples of this situation. This way, the general guideline applied in the catalogue is the identification of the landscape or area scale as an asset into the catalogue, and then the identification of those elements that have special classification circumstances through the classification criteria structure as new and different assets. Due to this, while each one of the elements that are included within that greater scale asset usually present a single new use (in cases in which they have been recovered for a new activity), the corresponding asset of the greater scale will consider the uses identified with all of them, although only one of them is in fact the use of that landscape or area. Thus, the presence of assets with a scale of landscape or area for social and cultural use is a consequence of the limitations of the classification criteria structure, not a real relation between that scale and that use.

In the case of "administrative and offices" use, a certain preference for the scale named "system or set of buildings" is shown, but a clear trend is not able to point out the relation between the needs of the use and the characteristics of the scale criterion. Furthermore, regarding "educational and research" use, the preference for this scale is very significant. This is because in this case, the relation between the needs of the use and the distinctive characteristics of each scale exists, since a main need of educational centers is playgrounds, as spaces that can be disposed between the buildings and elements of some typical industrial facilities layouts. These kinds of conclusions are of great value when multi-criteria decision structures for the selection of the most compatible new uses are built [1,19].

Finally, the third graph included in Figure 12 shows the scales linked to "monument or sculpture" use. This use is mainly focused on chimneys and other elements, such as furnaces, which are inhabitable due to their scale. Thus, this kind of asset does not have the capacity to accommodate any kind of new uses. Nevertheless, they are industrial elements of interest, so they must be identified, classified, and protected. In Figure 12, it is possible to appreciate the significant presence of this scale, but also the "immovable asset" scale has presence, especially in the province of Cádiz. This is due to the significant presence of windmills in this territory, which was previously exposed and also graphically illustrated in Figure 9.

New criteria added to the classification criteria structure of the catalogue can be also analyzed, both from approaches focused on their own information and combined with other criteria. As exposed before in the section "relation with [the] local population", these criteria try to show the possible synergies between each asset and the nearest population centers. Two main aspects affect this relation: the population of the corresponding village, town, or city, and the road communication and general accessibility to the asset from the corresponding population center. Both aspects are important when new uses are proposed, but a connection between both elements is the key issue. A large population provides potential users with the new activities, but if there is no real relation between the asset and the population center, its size will not be relevant. This idea is graphically explained in Figure 6. Thus, six relation criteria categories are defined, which can be understood as seven when the special role of "province capital" exists. Firstly, Figure 13 analyses the size of the nearest population centers for each asset of the catalogue. 

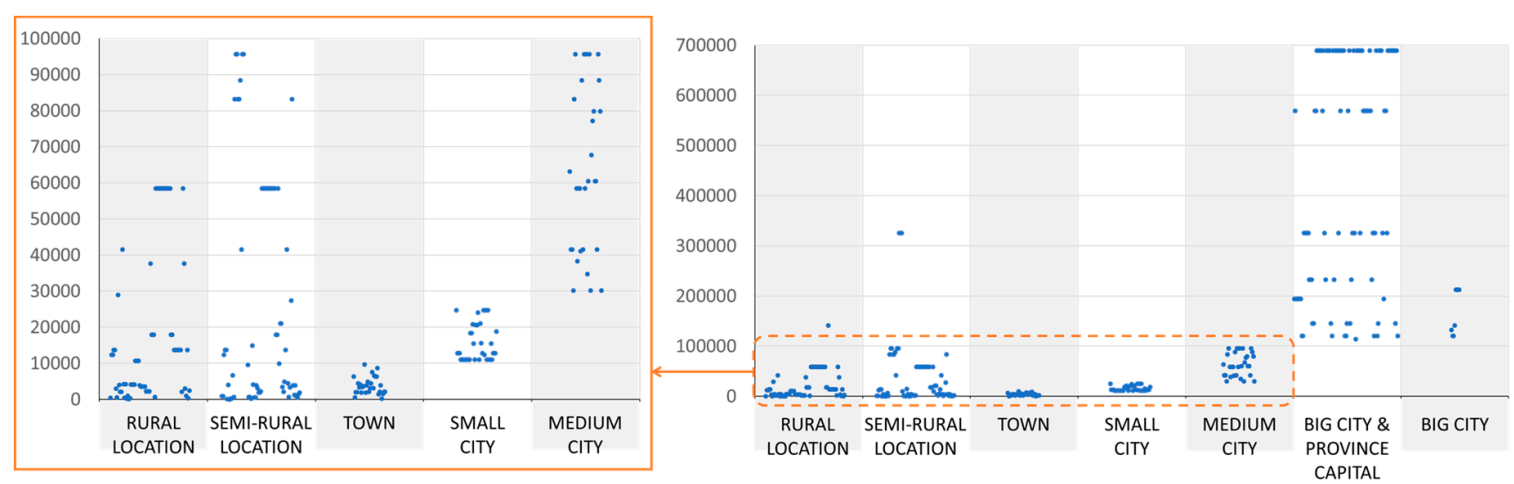

Figure 13. Distribution of assets within classification categories focused on the relationships with population centers by considering the number of inhabitants.

As exposed in Figure 6, three main situations can be distinguished by considering the relation between the asset and the nearest center of population; rural locations, semi-rural locations, and city or urban locations. Within urban contexts, four subcategories are defined (town, small city, medium city, and big city), making it possible to distinguish different sizes. Size is a key aspect to understand how a city works. Road and communication systems, infrastructures, and services are examples of aspects with different dimensions and complexity levels according to the type of city. However, rural and semi-rural locations have no relation to the size of the nearest population centers. In that sense, Figure 13 analyses the different sizes identified for the assets classified within each one of the categories that were considered.

It is possible to appreciate how population centers that are linked to assets with rural and semi-rural locations have in many cases significant sizes, which are similar to the ones identified for small and medium cities. On the left side of Figure 13, a zoom of that part of the graph is made in order to appreciate these situations more easily. The real potential synergies with population centers when a possible new activity is evaluated for an asset will be conditioned by aspects such as the size of those villages, towns, and cities, so these approaches are of interest when reutilization studies are being developed. On the other hand, Figure 13 reveals how most of the assets that have been identified for the category of big cities are located in province capitals, although there are a few located in some of the other big cities identified in Table 2. Despite the size of the cities, their social and administrative roles are also important, so not only the size is relevant, but also being the capital of a region has a great value, and can make it easier to develop these kinds of reuse projects.

In that sense, Figure 14 tries to identify the relations between these location categories and the presence of reuse initiatives. As it is shown, there is a great leap in this aspect between rural and semi-rural locations and the rest of the categories, which are associated with urban contexts. This highlights the influence of context circumstances for the success of reuse actions, but the identification of those examples that exists in rural and semi-rural environments and the strategies applied within them are also interesting.

Within the urban categories, the highest reuse values are identified for bigger city sizes. Thus, medium and large cities are the categories with a greater percentage of assets reused for new activities. In the case of big cities, the role as a province capital is able to increase this percentage, but not in a really significant way. It is also interesting that medium-size cities obtain a major reuse percentage. This can be due to the more aggressive urban growth processes that usually happen in big cities and which significantly modify the uses and the land value, which are aspects that make the conservation of old industrial facilities very complicated from a business viewpoint. In addition, the bigger the city is, the more difficult the feelings of belonging to the social history and tradition of the place among the population. These feelings are able to contribute to the conservation of the elements of this typology in a key way, or not. 


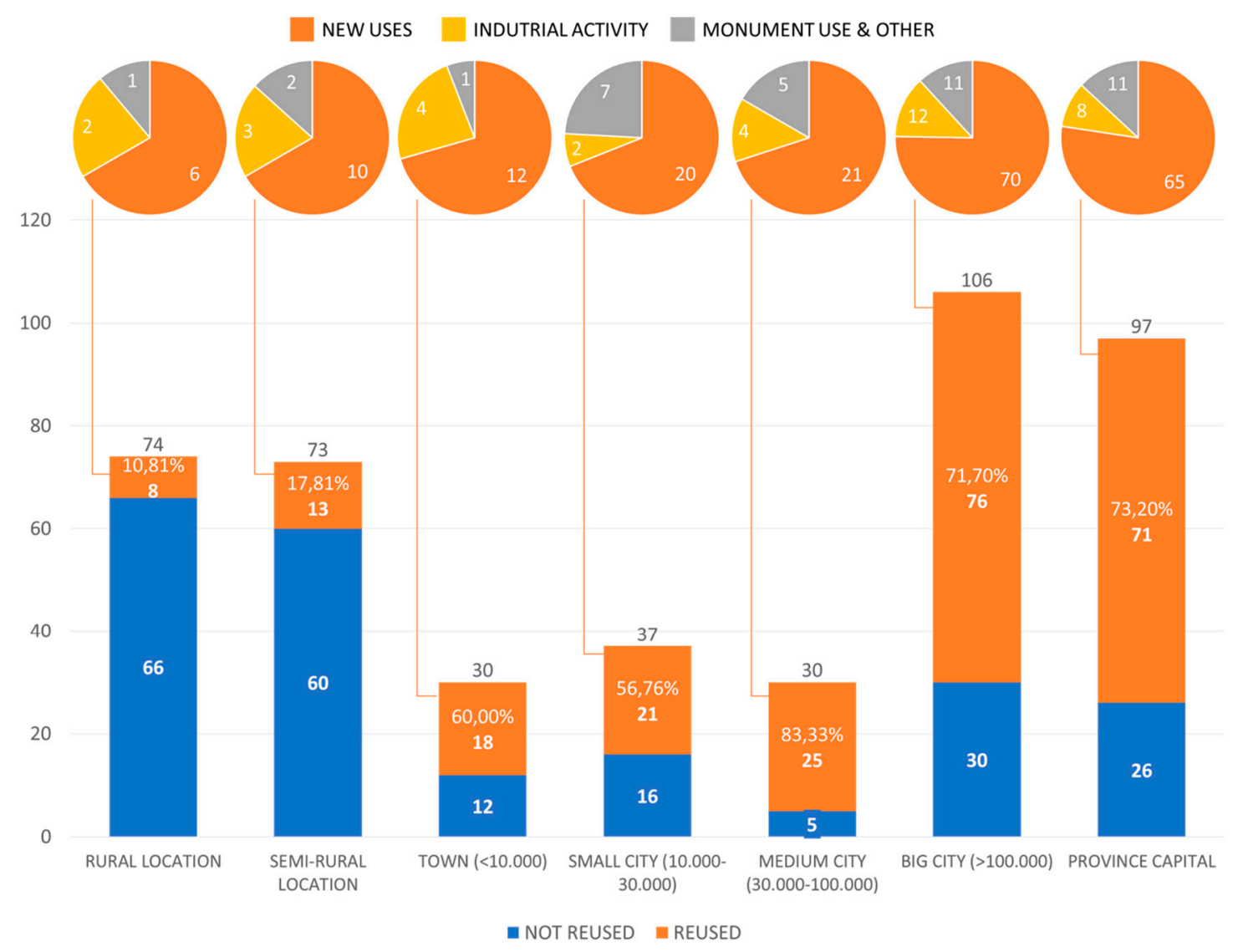

Figure 14. Assets reused and not reused for each classification category referred to the relation with the local population. New uses are of a general nature.

\section{Conclusions}

The analyses shown in this work are just some of the possible approaches that the developed catalogue allows, but they are considered as representative of the possibilities and potential that these kinds of tools can have in the study, promotion, management, and protection of the assets of the industrial heritage. The results obtained are considered of interest for all of these tasks.

The importance of the incorporation of sufficiently complete criteria structures to the cataloguing initiatives is a key aspect for their usefulness. Industrial heritage cannot be promoted or studied through simple compilations of assets. Their nature and main characteristics must be ordered through an appropriate classification criteria structure that is able to allow both the characterization of the typology and the contextualization of each asset within the sample. This work defines and proposes a full criteria structure for the classification of this type of assets. The main aspects to be taken into account when industrial assets are analyzed are studied through different groups of criteria that are focused on each one of those key aspects. Application guidelines are also given in those cases in which more doubts can rise, what is critical if the catalogue wants to be understood as a real tool.

Thus, the authors consider the developed catalogue as a useful tool in the field of the industrial heritage, both in the international context as a methodological approach and in the particular context of Spain as a tool that represents a significant progress from the previous situation. This usefulness is highlighted in the other works of the authors in which the catalogue and the criteria structure applied to all of them is the substrate in which other methodologies are based [1,19]. Indeed, the new criteria have been included in the structure of the catalogue as a response to the needs that have been identified during its use.

Regarding further developments of the catalogue, and particularly with regard to the actualization of the sample in other territories and the continue revision of the full set of assets considered, the most 
valuable strategy is a collaborative growth of the catalogue. The authors have previous experiences in the collaborative cataloguing of industrial assets within a university context with positive results. Thus, transforming the catalogue developed in an open tool represents an interesting challenge not only for social promotion of the industrial heritage in Spain, but also as a growth strategy of the sample. Probably, a collaborative identification of assets is the only sustainable and successful strategy for the development of a complete catalogue of this typology in such a large territory as Spain. By availing the knowledge of the potential users of the catalogue located throughout the national territory, which are who know the best examples in their territories, it would be possible to identify and include a large number of assets of great value.

Author Contributions: Conceptualization, J.C. and M.A.S.; methodology, J.C. and M.A.S.; software, J.C. and A.G.-D.; validation, J.C., A.G.-D., L.S. and M.A.S.; formal analysis, J.C., A.G.-D. and L.S.; investigation, J.C. and A.G.-D., L.S. and M.A.S.; resources, J.C., L.S. and M.A.S.; data curation, J.C., A.G.-D., L.S. and M.A.S.; writing-original draft preparation, J.C. and A.G.-D.; writing-review and editing, J.C., A.G.-D., L.S. and M.A.S.; visualization, J.C. and A.G.-D.; supervision, L.S. and M.A.S.; project administration, J.C. and M.A.S.; funding acquisition, J.C., L.S. and M.A.S.

Funding: This research was partly funded by the Manufacturing Engineering Society (http:/ /www.sif-mes.org/) through a mobility grant given to the first author. This research period was developed in the University of Malaga during 2016 and supervised by Professor Sevilla and Professor Sebastián. The results obtained during this research stay represent a significant part of the support data used in this work.

Conflicts of Interest: The authors declare no conflict of interest.

\section{References}

1. Claver, J. Methodology for the Analysis and Interpretation of the Assets of the Spanish Industrial Heritage. Application to the Study of the Assets of the Autonomous Community of Madrid. Ph.D. Thesis, Universidad Nacional de Educación a Distancia, Madrid, Spain, 27 April 2016.

2. Institute of the Cultural Heritage of Spain (IPCE), Ministry of Culture and Sport, Spanish Government. National Plan of Industrial Heritage. Available online: http://www.mecd.gob.es/planes-nacionales/ dam/jcr:eba404cd-e170-419d-b46a-e241ebd1b1b0/04-texto-2016-pnpi-plan-y-anexos.pdf (accessed on 11 September 2018).

3. Biel, P.; Cueto, G. 100 Elementos del Patrimonio Industrial en España; TICCIH-IPCE-CICEES: Madrid, Spain, 2009; 324p, ISBN 978-84-937738-6-1.

4. Tostóes, A.; García, C.; Landrove, S. La Arquitectura de la Industria, 1925-1965; Registro DOCOMOMO Ibérico; DOCOMOMO Ibérico Foundation: Barcelona, Spain, 2005; 276p, ISBN 84-609-1196-9.

5. The Declaration of Amsterdam. Adopted at the Congress on the European Architectural Heritag; The Declaration of Amsterdam: Amsterdam, The Netherlands, 1975.

6. TICCIH. The Nizhny Tagil Charter for the Industrial Heritage; Adopted at the Triennial National Assembly of TICCIH; TICCIH: Moscow, Russia, 2003.

7. Llamas, J.; Lerones, P.M.; Medina, R.; Zalama, E.; Gómez-García-Bermejo, J. Classification of Architectural Heritage Images Using Deep Learning Techniques. Appl. Sci. 2017, 7, 992. [CrossRef]

8. Belhi, A.; Bouras, A.; Foufou, S. Leveraging Known Data for Missing Label Prediction in Cultural Heritage Context. Appl. Sci. 2018, 8, 1768. [CrossRef]

9. Oglethorpe, M.; McDonald, M. Recording and documentation. In Industrial Heritage Re-Toleed; Douet, J., Ed.; Routledge: New York, NY, USA, 2013; pp. 55-62.

10. Rossnes, G. Process recording. In Industrial Heritage Re-Toleed; Douet, J., Ed.; Routledge: New York, NY, USA, 2013; pp. 63-69.

11. Claver, J.; Sebastián, M.A. Methodological study and characterization of the industrial heritage of the Autonomous Community of Galicia. Procedia Manuf. 2017, 13, 1305-1311. [CrossRef]

12. Claver, J.; Sebastián, M.A.; Marcos-Bárcena, M.; Sevilla, L. Identification and characterization of the industrial heritage of the Province of Cádiz. Application of methodologies of identification and classification of assets. In Proceedings of the 24th CUIEET, Cádiz, Spain, 21-23 September 2016. 
13. Claver, J.; Sebastián, M.A. Methodological proposes for the study of industrial assets. Application to the Province of Huelva. In Proceedings of the 1st International Congress on Industrial Heritage and Public Work, Huelva, Spain, 26-28 October 2016.

14. Claver, J.; Sebastián, M.A. Methodological analysis of the assets of the industrial heritage in the Autonomous Community of the Region of Murcia. In Proceedings of the 20th International Congress on Project Management and Engineering, Cartagena, Spain, 13-15 July 2016.

15. Claver, J.; Sebastián, M.A.; Sevilla, L. Methodology for the study of the industrial heritage. Application to the Autonomous Community of Andalusia. DYNA 2016, 91, 136-139. [CrossRef]

16. Rojas-Sola, J.I.; Castro-García, M.; Carranza-Canadas, M.P. Content Management System incorporated in a virtual museum hosting. J. Cult. Herit. 2011, 12, 74-81. [CrossRef]

17. Rojas-Sola, J.I.; Gómez, M.C.; Castro, M. Windmills in Andalusia: new tools for their valorization. Asociación de Geógrafos Españoles 2013, 62, 403-427. [CrossRef]

18. Vacca, G.; Fiorino, D.R.; Pili, D. A Spatial Information System (SIS) for the Architectural and Cultural Heritage of Sardinia (Italy). ISPRS Int. J. Geo-Inf. 2018, 7, 49.

19. Claver, J.; García-Domínguez, A.; Sebastián, M.A. Decision-Making Methodologies for Reuse of Industrial Assets. Complexity 2018, 2018, 4070496. [CrossRef]

20. Nocca, F. The Role of Cultural Heritage in Sustainable Development: Multidimensional Indicators as Decision-Making Tool. Sustainability 2017, 9, 1882. [CrossRef]

21. Liu, F.; Zhao, Q.; Yang, Y. An approach to assess the value of industrial heritage based on Dempster-Shafer theory. J. Cult. Herit. 2018, 32, 210-220. [CrossRef]

22. Giuliani, F.; De Falco, A.; Landi, S.; Bevilacqua, M.G.; Santini, L.; Pecori, S. Reusing grain silos from the 1930s in Italy. A multi-criteria decision analysis for the case of Arezzo. J. Cult. Herit. 2018, 29, 145-159. [CrossRef]

23. National Institute of Statistical of Spain (INE). Demography and Population Database. 2017. Available online: https: / / www.ine.es/dyngs/INEbase/es/categoria.htm?c=Estadistica_P\&cid=1254734710984 (accessed on 28 October 2018).

24. Google Maps. Available online: https:/ / www.google.com/maps (accessed on 10 January 2018).

(C) 2019 by the authors. Licensee MDPI, Basel, Switzerland. This article is an open access article distributed under the terms and conditions of the Creative Commons Attribution (CC BY) license (http:/ / creativecommons.org/licenses/by/4.0/). 